\title{
Terahertz frequency receiver instrumentation for Herschel's heterodyne instrument for far infrared (HIFI)
}

John C. Pearson, Imran Mehdi, Erich Schlecht, Frank Maiwald, Alain Maestrini, et al.

John C. Pearson, Imran Mehdi, Erich Schlecht, Frank Maiwald, Alain Maestrini, John J. Gill, Suzanne C. Martin, Dave Pukala, J. Ward, Jonathan Kawamura, William R. McGrath, William Hatch, Dennis G. Harding, Henry G. LeDuc, Jeffry A. Stern, Bruce Bumble, Lorene A. Samoska, Todd C. Gaier, Robert Ferber, David Miller, Alexandre Karpov, Jonas Zmuidzinas, Thomas G. Phillips, Neal R. Erickson, Jerry Swift, Yun Chung, Richard Lai, Huei Wang, "Terahertz frequency receiver instrumentation for Herschel's heterodyne instrument for far infrared (HIFI)," Proc. SPIE 4850, IR Space Telescopes and Instruments, (5 March 2003); doi: 10.1117/12.461757 


\title{
THz Frequency Receiver Instrumentation for Herschel's Heterodyne Instrument for Far Infrared (HIFI)
}

\author{
J.C. Pearson ${ }^{* a}$, I. Mehdia , E. Schlecht ${ }^{\mathrm{a}}$, F. Maiwald ${ }^{\mathrm{a}}$, A. Maestrini ${ }^{\mathrm{a}}$, J. Gill ${ }^{\mathrm{a}}$, S. Martin ${ }^{\mathrm{a}}$, D. Pukala ${ }^{\mathrm{a}}$, J. \\ Ward $^{\mathrm{a}}$, J. Kawamura ${ }^{\mathrm{a}}$, W.R. McGrath ${ }^{\mathrm{a}}$, W.A. Hatch ${ }^{\mathrm{a}}$, D. Harding ${ }^{\mathrm{a}}$, H.G. Leduc ${ }^{\mathrm{a}}$, J.A. Stern ${ }^{\mathrm{a}}$, B. \\ Bumble $^{\mathrm{a}}$, L. Samoska ${ }^{\mathrm{a}}$, T. Gaier ${ }^{\mathrm{a}}$, R. Ferber ${ }^{\mathrm{a}}$, D. Miller ${ }^{\mathrm{b}}$, A. Karpov ${ }^{\mathrm{b}}$, J. Zmuidzinas ${ }^{\mathrm{b}}$, T. Phillips ${ }^{\mathrm{b}}$, N. \\ Erickson $^{\mathrm{c}}$, J. Swift ${ }^{\mathrm{d}}$, Y.-H. Chung ${ }^{\mathrm{d}}$ R. Lai ${ }^{\mathrm{d}}$, and H. Wang ${ }^{\mathrm{e}}$
}

${ }^{a}$ Jet Propulsion Laboratory, California Institute of Technology, Mail Stop 301-429, 4800 Oak Grove Dr., Pasadena, CA 91109, ${ }^{\mathrm{b}}$ California Institute of Technology, Mail Stop 320-47, Pasadena, CA 91125, ${ }^{\mathrm{C}}$ University of Massachusetts Amherst, Amherst MA 01035, ${ }^{\mathrm{d}} \mathrm{TRW}$, Inc., One Space Park, Redondo Beach CA 90278, ${ }^{\mathrm{e}}$ National Taiwan University, Taipei Taiwan ROC

\begin{abstract}
The Heterodyne Instrument for Far Infrared (HIFI) on ESA's Herschel Space Observatory is comprised of five SIS receiver channels covering 480-1250 GHz and two HEB receiver channels covering 1410-1910 GHz. Two fixed tuned local oscillator sub-bands are derived from a common synthesizer to provide the front-end frequency coverage for each channel. The local oscillator unit will be passively cooled while the focal plane unit is cooled by superfluid helium and cold helium vapors. HIFI employs W-band GaAs amplifiers, InP HEMT low noise IF amplifiers, fixed tuned broadband planar diode multipliers, and novel material systems in the SIS mixers. The National Aeronautics and Space Administration's Jet Propulsion Laboratory is managing the development of the highest frequency $(1119-1250 \mathrm{GHz})$ SIS mixers, the highest frequency $(1650-1910 \mathrm{GHz})$ HEB mixers, local oscillators for the three highest frequency receivers as well as W-band power amplifiers, varactor diode devices for all high frequency multipliers and InP HEMT components for all the receiver channels intermediate frequency amplifiers. The NASA developed components represent a significant advancement in the available performance. The current state of the art for each of these devices is presented along with a programmatic view of the development effort.
\end{abstract}

Keywords: Heterodyne, Receiver, Submillimeter, Multiplier, Mixer, LNA, Local Oscillator, MMIC, THz

\section{INTRODUCTION}

The desired functionality and performance of the Heterodyne Instrument for Far Infrared (HIFI) scheduled for launch on ESA's Herschel Space Observatory (Herschel) required a number of significant advances in heterodyne instrumentation over previous generations of ground based heterodyne receivers. The driving requirement is that the HIFI instrument be an observatory class facility instrument with sufficient flexibility to support a large number of observing modes and observational programs at previously unattainable frequencies and with nearly complete frequency coverage. These requirements must be achieved in a largely autonomous space based environment. This paper presents a general overview of the entire program of the HIFI "High Frequency Subsystem" papers $4855-45^{1}, 4855-50^{2}, 4855-55^{3}, 4855$ $56^{4}, 4855-58^{5}, 4855-60^{6}$ and $4855-61^{7}$ in the conference on Millimeter and Submillimeter Detectors present more detail on the individual technologies introduced here.

The HIFI instrument is a seven-channel, single-pixel, dual polarization, double sideband receiver system with one receiver operated at time with a 4 to $8 \mathrm{GHz}$ intermediate frequency analyzed separately in both E- and H-Plane polarizations by two $4 \mathrm{GHz}$ bandwidth array acousto-optical spectrometers and two higher resolution narrower banded autocorrelation spectrometers. Further details on the HIFI instrument capabilities are presented in paper $4850-95^{8}$.

\footnotetext{
* Send correspondence to John.C.Pearson@jpl.nasa.gov
} 
The spectrometers performance and capabilities are discussed in a previous SPIE Proceeding ${ }^{9,10}$. Table 1 provides a frequency table of the HIFI receiver bands and denotes the mixer and local oscillator bands. Details of the local oscillator system have been discussed previously ${ }^{11}$ and are updated in paper 4850-97 ${ }^{12}$. The local oscillator requires fixed tuned multipliers covering the nominal bands in table 1 .

Table 1 Frequency bands of HIFI

\begin{tabular}{|l|l|l|l|l|l|l|}
\hline & Frequency & E-Mixer & H-Mixer & IF & LO a & LO b \\
\hline Band 1 & $480-641$ & SIS & SIS & 4 to 8 & $488-546$ & $560-633$ \\
\hline Band 2 & $639-801$ & SIS & SIS & 4 to 8 & $647-710$ & $724-793$ \\
\hline Band 3 & $799-961$ & SIS & SIS & 4 to 8 & $807-848$ & $862-953$ \\
\hline Band 4 & $959-1121$ & SIS & SIS & 4 to 8 & $967-1042$ & $1056-1113$ \\
\hline Band 5 & $1119-1250$ & SIS & SIS & 4 to 8 & $\mathbf{1 1 2 7 - 1 1 7 8}$ & $\mathbf{1 1 9 2 - 1 2 4 2}$ \\
\hline Band 6L & $1410-1704$ & NbN HEB & NbN HEB & 4 to 8 & $\mathbf{1 4 1 8 - 1 5 4 0}$ & $\mathbf{1 5 5 4 - 1 6 9 6}$ \\
\hline Band 6H & $1702-1910$ & Nb HEB & Nb HEB & 4 to 8 & $\mathbf{1 7 1 0 - 1 8 0 0}$ & $\mathbf{1 8 1 4 - 1 9 0 2}$ \\
\hline
\end{tabular}

Bold= NASA/JPL Supplied Subsystem

Italics $=$ NASA/JPL Supplied Components

\section{SCIENCE REQUIREMENTS}

The HIFI instrument is designed to facilitate an observatory class set of science requirements. The major goal of the instrument is to observe the cold molecular clouds and dust occupying much of our galaxy and distant galaxies at frequencies impossible for ground based instrumentation. As a result, HIFI requires the best sensitivity possible, which translates into high optical throughput, cold optics and the lowest possible mixer and intermediate frequency amplifier noise temperatures. The emphasis is on the water frequencies between $480 \mathrm{GHz}$ and $980 \mathrm{GHz}$ and coverage above 980 $\mathrm{GHz}$ where observations are nearly impossible from the ground. The velocity distribution in a spatially unresolved galaxy require an instantaneous IF bandwidth of approximately $4 \mathrm{GHz}$ as does the desire to make comprehensive spectral surveys of objects with relatively small amounts of observing time. Lastly the red shifts of strong lines in distant objects must be compensated for as well as separation of upper and lower sideband components, requiring the local oscillator to have a small frequency step size and nearly complete frequency coverage.

In order to observe the cold universe, HIFI requires the highest sensitivity possible mixer at all frequencies. Limitations in local oscillator power bandwidth at high frequencies require the mixers to operate with low levels of local oscillator power. The combination of sensitivity, bandwidth and limited local oscillator power lead to the use of cryogenic superconductor insulator superconductor (SIS) or hot electron bolometer (HEB) mixers in conjunction with diplexer local oscillator injection. Ultimate sensitivity and stored cryogens required the IF amplifiers to have very low noise and very low power dissipation. For observatory operation the local oscillator needed full frequency coverage and no mechanical tuning elements. At the time of the HIFI proposal none of these requirements had been full demonstrated in the necessary combination and significant development especially in the local oscillator was required.

\section{MIXERS}

The HIFI instrument and science objectives required the extension of SIS mixer technology above the $\mathrm{Nb}$ superconducting band gap of $750 \mathrm{GHz}$ in four of the HIFI SIS bands. The defined bands also required a departure from the single junction mixer with $\mathrm{AlO}_{\mathrm{x}}$ barriers, which are limited to about $120 \mathrm{GHz}$ of bandwidth by the practically obtainable ratio of tunneling to capacitance in the junction. The HIFI instruments bands above $1.4 \mathrm{THz}$ required the perfection new HEB mixers. Extra galactic science objectives required the HEB mixers to have a wide IF bandwidth.

Prior to the HIFI project start in late 1997, the state of the art mixers were Nb SIS devices, which were limited to low noise operation below the $\mathrm{Nb}$ superconducting band gap of approximately $750 \mathrm{GHz}$. The bandwidth was also limited to approximately $120 \mathrm{GHz}$ by the ratio of the tunneling to capacitance in the junction. A couple of experiments had demonstrated the use of $\mathrm{Nb} / \mathrm{AlOx} / \mathrm{Nb}$ junctions with normal metal ground planes and tuning structures proving the feasibility of making mixers to twice the superconducting band gap ${ }^{13,14}$. Both phonon and diffusion cooled HEB mixers 
had recently been demonstrated in the laboratory ${ }^{15-17}$, but neither had demonstrated the desired IF bandwidth and there were only one project in progress to use a phonon-cooled device outside the laboratory.

The ultimate low noise SIS mixer performance requires the use of a superconducting tuning circuit with a band gap above the operation frequency, which required mixers made from new materials. The HIFI bands large bandwidth required multiple junctions or a larger tunneling to capacitance ratio than had previously been achieved. Additionally, the HIFI band 5 mixers upper frequency of $1250 \mathrm{GHz}$ was uncomfortably close to twice the band gap of $\mathrm{Nb}$ so a larger band gap superconductor was desirable in one or both junction electrodes. The first major breakthrough came with the successful implementation of $\mathrm{NbTiN}$ in the junction, wiring and ground plain of an SIS mixer ${ }^{18}$. An $800 \mathrm{GHz}$ mixer with an NbTiN/AlN/Nb junction, NbTiN wiring and NbTiN ground plane was installed as a facility receiver at the Caltech Submillimeter Observatory with a Trec of $205 \mathrm{~K}^{19}$. In the process of experimenting with the AlN barriers it was discovered that very high current density $>100 \mathrm{kA} \mathrm{cm}^{-1}$ could be fabricated and that junctions with $30 \mathrm{kA} \mathrm{cm}^{-1}$ and large subgap-to-normal state resistance ratios could be fabricated reliabily ${ }^{20}$. The development effort was dealt a setback when, a heterodyne response was not observed in a $1200 \mathrm{GHz}$ SIS mixer with a NbTiN ground plane mixer in spite of an FTS response. The reason the heterodyne response was not observed is still under investigation, but is believed to be due to skin effects and surface layers in NbTiN. The fall back, which was adopted, was to continue with the high current density $\mathrm{Nb} / \mathrm{AlN} / \mathrm{NbTiN}$ junction, minimize the importance of the tuning structure by reducing the resistance of the normal metal ground plane and wiring layers. It was subsequently discovered that in thin films the largest reduction in resistance and subsequent lowest resistance at cryogenic temperatures could be obtained with epitaxial $\mathrm{Nb}^{21}$.

The baseline HIFI band 5 mixers use $\mathrm{Nb} / \mathrm{AlN} / \mathrm{NbTiN}$ junctions normal metal wire and $\mathrm{Nb}$ ground planes on sapphire substrates with an integrated twin slot antennas shown in Figure 1a. The mixer devices are integrated in a quasi-optical mount with a $5 \mathrm{~mm}$ diameter lens shown in figure $1 \mathrm{~b}$. Receiver temperatures have consistently been under $1000 \mathrm{~K}$ in air without correction for windows, beam splitters or atmospheric attenuation. Current efforts are focused on understanding the high frequency physics details in order to achieve some improvement prior to flight delivery in 2003. Further details on the HIFI band 5 mixer development and device physics will be presented in paper $4855-50^{2}$.

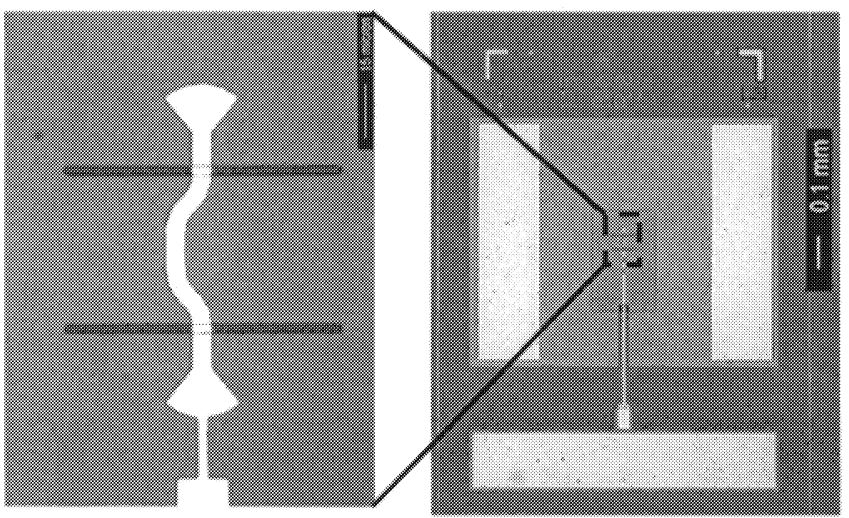

Figure 1a

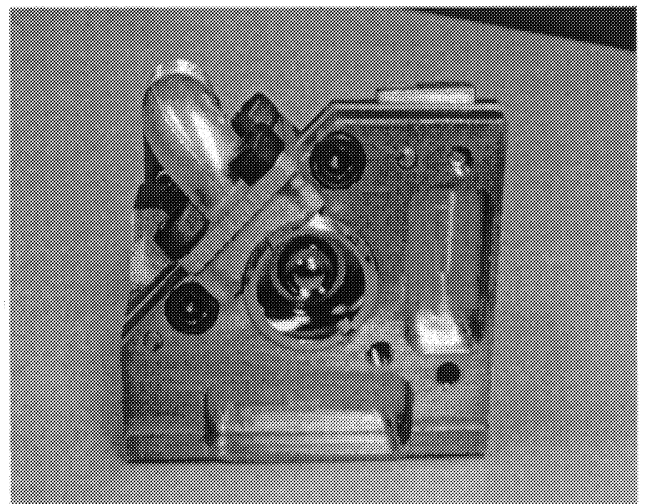

Figure $1 \mathrm{~b}$

Figure 1a: Shows the SIS device and an expanded view of the twin junction twin slot mixer circuit. Figure 1b: Shows a prototype HIFI band 5 mixer without its cover. The width and height are within the assigned $32 \mathrm{~mm}$ envelope.

The development of HIFI HEB mixers was originally driven by technical concerns over the amount of available local oscillator power at $\mathrm{THz}$ frequencies. As a result, a major program was undertaken to develop Al HEB mixers, which promised wide IF bandwidths due to large diffusion constants and miniscule local oscillator power requirements due to the scaling of local oscillator power with the critical temperature squared. These predictions were subsequently demonstrated with laboratory measurements where Al HEB mixers repeatedly demonstrated local oscillator needs of less than $5 \mathrm{nW}$ absorbed, IF bandwidths of more $3 \mathrm{GHz}$ in 1 micron long devices and comparable noise and conversion efficiency to $\mathrm{Nb}$ devices ${ }^{22-24}$. In spite of encouraging performance the $\mathrm{Al}$ HEB devices saturated at very low levels of input making implementation in a real world receiver system problematic ${ }^{22,24}$. 
A parallel development in the $\mathrm{Nb}$ devices demonstrated a $9 \mathrm{GHz} 3 \mathrm{~dB}$ bandwidth in a 0.1 micron long device and noise temperature of under $2000 \mathrm{~K}$ at $2.5 \mathrm{THz}^{25}$. The IF bandwidth and helium temperature operation of the $\mathrm{Nb}$ devices allowed HIFI to eliminate an extra lower frequency diplexer design, an extra IF amplifier design, two extra RF cables to the cryostat and two additional IF processors. The greatest progress in the Nb HEB mixer design has come in the area of junction fabrication repeatability, ESD survivability and in understanding the reliability. Figure 2 shows a current $\mathrm{Nb}$ HEB with an integrated NbTiN protection circuit. The NbTiN has a resistance of several thousand ohms at room temperature and zero below about $10 \mathrm{~K}$. This series resistance results in approximately a 50 to 1 voltage division at the HEB element during room temperature handling, which raised the human body model transient the device could withstand to about 50 volts. The device shelf-life has been characterized for oxygen, humidity and temperature exposure. Two separate activation energies were found suggesting several chemical processes are at work in the device. The shelflife is sufficient for long integrations associated with cryogenic space missions, but the devices do degrade with time, temperature and exposure to humidity or oxygen. The HEB devices still lack a comprehensive noise theory equivalent to Tucker theory in SIS mixers and a significant effort was put into Al devices, so the only major improvement in the devices has been the de-imbedding of the device impedance from the rest of the cirucit ${ }^{26,27}$. Paper $4855-45^{1}$ discusses the current status of $\mathrm{Nb}$ HEB development in more detail.

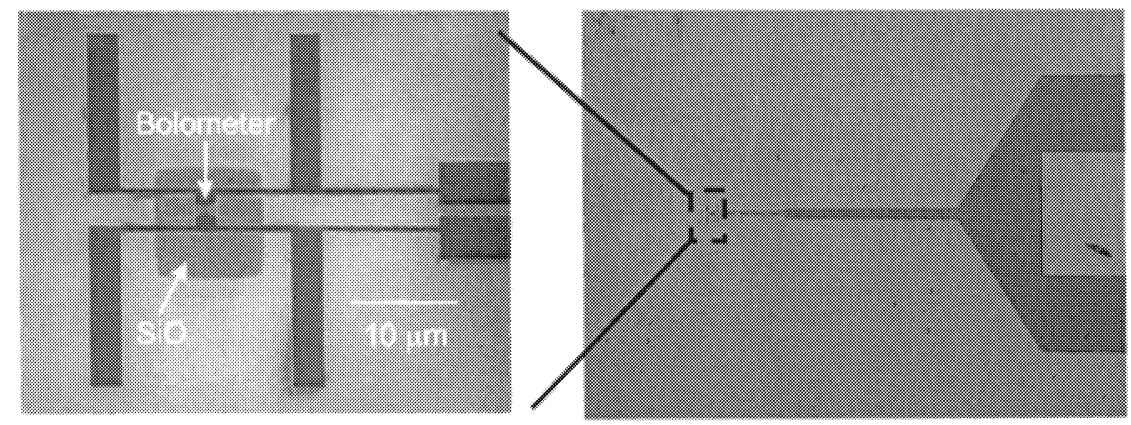

Figure 2. Nb HEB device and a scanning electron micrograph of the passivation and micro-bridge detail. The darker strip in the bias/IF line on the right is the NbTiN used as a protection resistor. The rest of the metalization is gold.

\section{AMPLIFIERS}

A consequence of low local oscillator power and highly sensitive cryogenic mixer operation is very low output noise at the IF of the mixer. As a result, the IF amplifier must have significantly lower noise than the mixer or the IF amplifier dominates the receiver noise. Low noise amplifiers fabricated from GaAs high electron mobility transistors (HEMTs) have been known for some time, however the limited supply of helium in Herschel requires lower power dissipation that GaAs and the quality of the HIFI mixers demand the best possible noise performance. InP devices can have higher gains and require lower bias voltages than GaAs. JPL and TRW have partnered with a number of ground based astronomy groups in a cryogenic HEMT optimization program. A large number of transistors and monolithic microwave integrated circuits have been produced using a TRW developed and optimized 0.1 micron gate InP process. This InP process has resulted in development of a number of remarkable low noise amplifiers with noise temperature in the 4-8 $\mathrm{GHz}$ band less than $4 \mathrm{Kelvin}^{6,7}$. A four-finger 200 micron gate length device made with TRW 0.1 micron InP process is shown in figure 3. The TRW process has demonstrated less than a microamp leakage in a 200 micron device and a transconductance greater than $1200 \mathrm{mS} / \mathrm{mm}$.

A pressing technical need of the HIFI instrument was to find an electrically tunable replacement for high power Gunn oscillators, which were rapidly becoming problematic to obtain space qualified and impossible to obtain with sufficient bandwidth to facilitate the local oscillator requirements. A potential solution was found in TRW's newly developed 0.1 micron gate $\mathrm{AlGaAs} / \mathrm{InGaAs} / \mathrm{GaAs}$ pseudomorphic T-gate power HEMT process on 50 micron substrates ${ }^{28}$. A TRW 94 $\mathrm{GHz}$ amplifier was tested for noise at the Caltech Submillimeter Observatory and shown to add no noise to the receiver relative to a Gunn oscillator ${ }^{29}$. A program was then undertaken to see if amplifiers with $15 \%$ bandwidth to $113 \mathrm{GHz}$ could be fabricated with the TRW process. Initially, coplanar waveguide low power amplifiers, microstrip driver 
amplifiers and power amplifiers of amplifiers were designed in three frequency bands 71-80, 89-105 and 100-113 $3^{30-32}$. Figure 4 shows a CPW pre-amplifier, a microstrip driver and a power amplifier.

The initial designs were able to achieve power levels greater than $200 \mathrm{~mW}$ at some frequencies, which was going to be marginal at the band edges for the HIFI local oscillators. Additionally HIFI required $>21 \mathrm{~dB}$ total gain, compressed operation to limit amplitude noise and $200 \mathrm{~mW}$ delivered to the multiplication chain. The result was a $+23.6 \mathrm{dBm}$ requirement at the $120 \mathrm{~K}$ operation temperature, which resulted in the need for a power combined module with multiple MMICs. Figure 5 shows the JPL designed evaluation and test module and schematic layout. A total of 12 power combined five amplifier modules and 5 three-stage modules have been assembled, tested and delivered for HIFI along with $>50$ additional single amplifiers. The five amplifier units have achieved $+29 \mathrm{dBm}$ at $95 \mathrm{GHz}$ when operated at $120 \mathrm{~K}$.

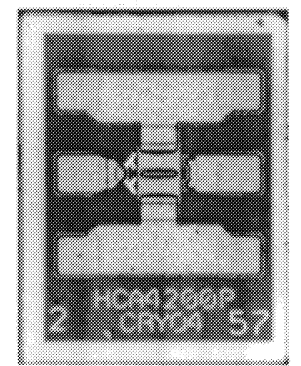

Figure 3. 0.1micron gate four-finger 200 micron gate length InP HEMT device from the cryogenic HEMT optimization program lot number 4 (cryo4).
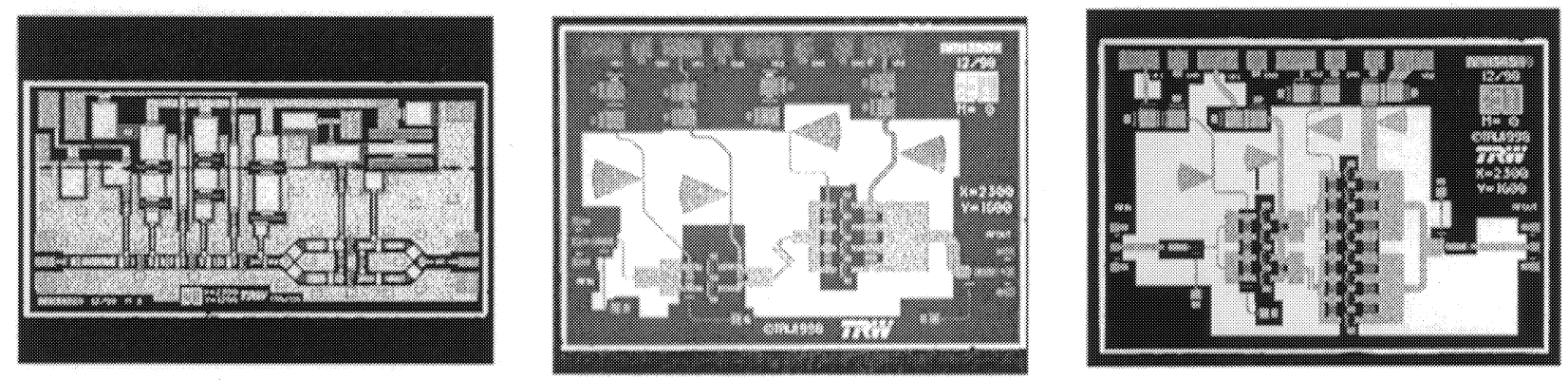

Figure 4. Power amplifier MMICs fabricated with TRW 0.1 micron GaAs Process, from left to right a CPW pre-amplifier, a microstrip driver and a microstrip power amplifier. The devices generate typical saturated power levels of $50 \mathrm{~mW}, 100 \mathrm{~mW}$ and $200 \mathrm{~mW}$, respectively.
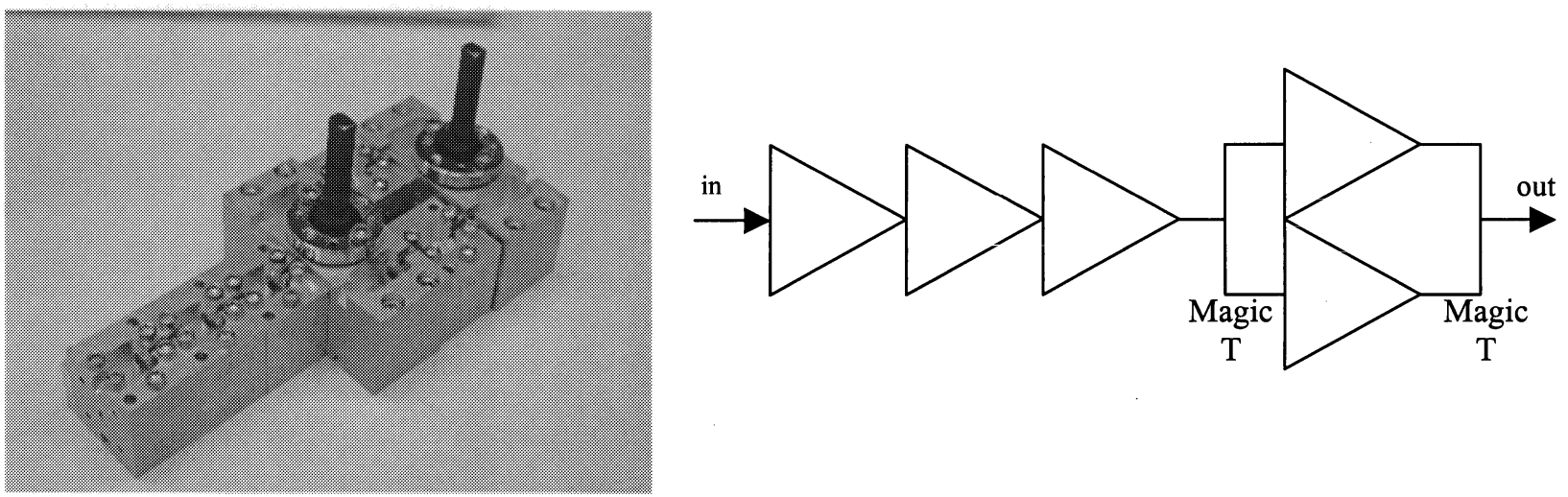

Figure 5. Development and test power amplifier module build from individual amplifiers and magic T's. Not shown is a 90 degree shim in front of one and behind the other output amplifiers making the package a 90 degree hybrid. 
Full evaluation of the prototype amplifiers identified a series of on MMIC problems. All the microstrip amplifiers oscillated at $\sim 32 \mathrm{GHz}$ and some oscillated at $47 \mathrm{GHz}$ as well. The problem was tracked to the odd mode suppression resistors between the arms of the on MMIC Wilkenson couplers. The causes and methods for evaluation and determining corrections have been presented in detail elsewhere ${ }^{33}$. The next task was to develop a flight module with a requirement for less than 6 watts power consumption and a mass goal of $100 \mathrm{~g}$. Figure 6 shows TRW's w-band module and the schematic configuration. This module achieves the required performance with worst-case power consumption of 5.5 Watts and is $101.5 \mathrm{~g}$. These modules give nearly identical performance to the prototypes when the parallel stages are well matched, however the use of two driver amplifiers rather than a power amplifier in the TRW packages give about 2 $\mathrm{dB}$ of extra gain. Figure 7 is typical power outputs of the power-combined modules with well-matched output stage MMICs. As can be seen in figure 6, the $92 \mathrm{GHz}$ end of the $80-92 \mathrm{GHz}$ amplifier, the $88 \mathrm{GHz}$ end of the 88-99 amplifier and the $106 \mathrm{GHz}$ end of the 92-106 amplifier have less margin than desirable. As a result, the driver and power amplifier MMICs in each of these bands are being modified to give slightly better performance at the band edges. The TRW module has survived the qualification level 60 thermal cycles to 120 Kelvin with no degradation. Additional details on this technology are presented in $4855-58^{5}$.
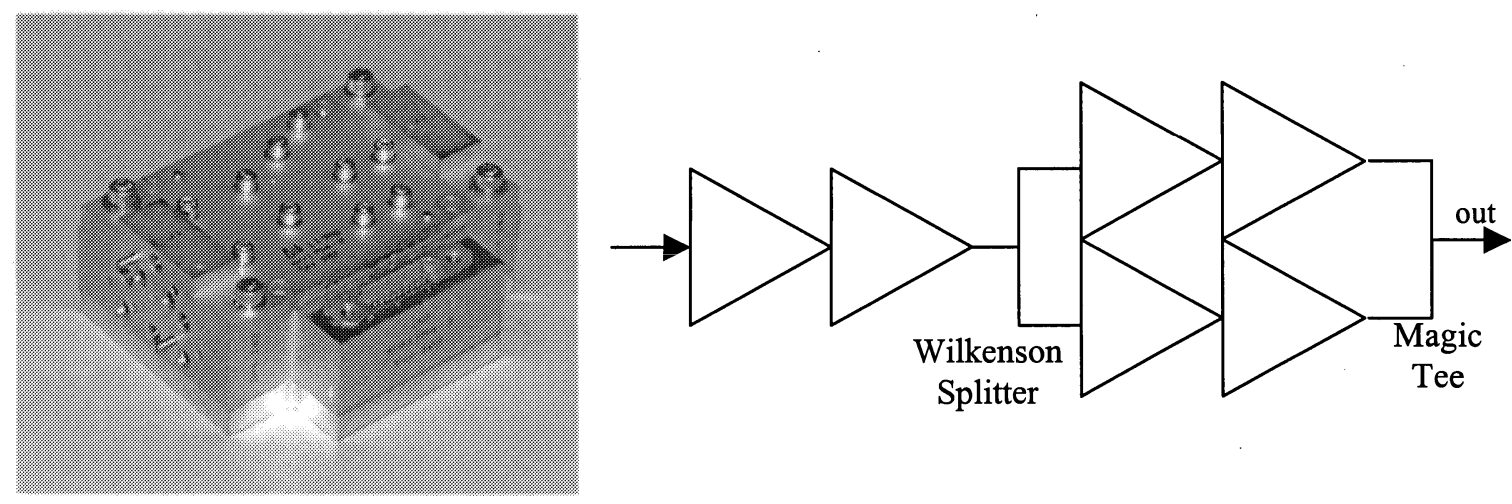

Figure 6 Shows the TRW Herschel HIFI power amplifier module and its schematic configuration. Not shown is a 90 degree shim before one of the parallel arms and after the other parallel arm.

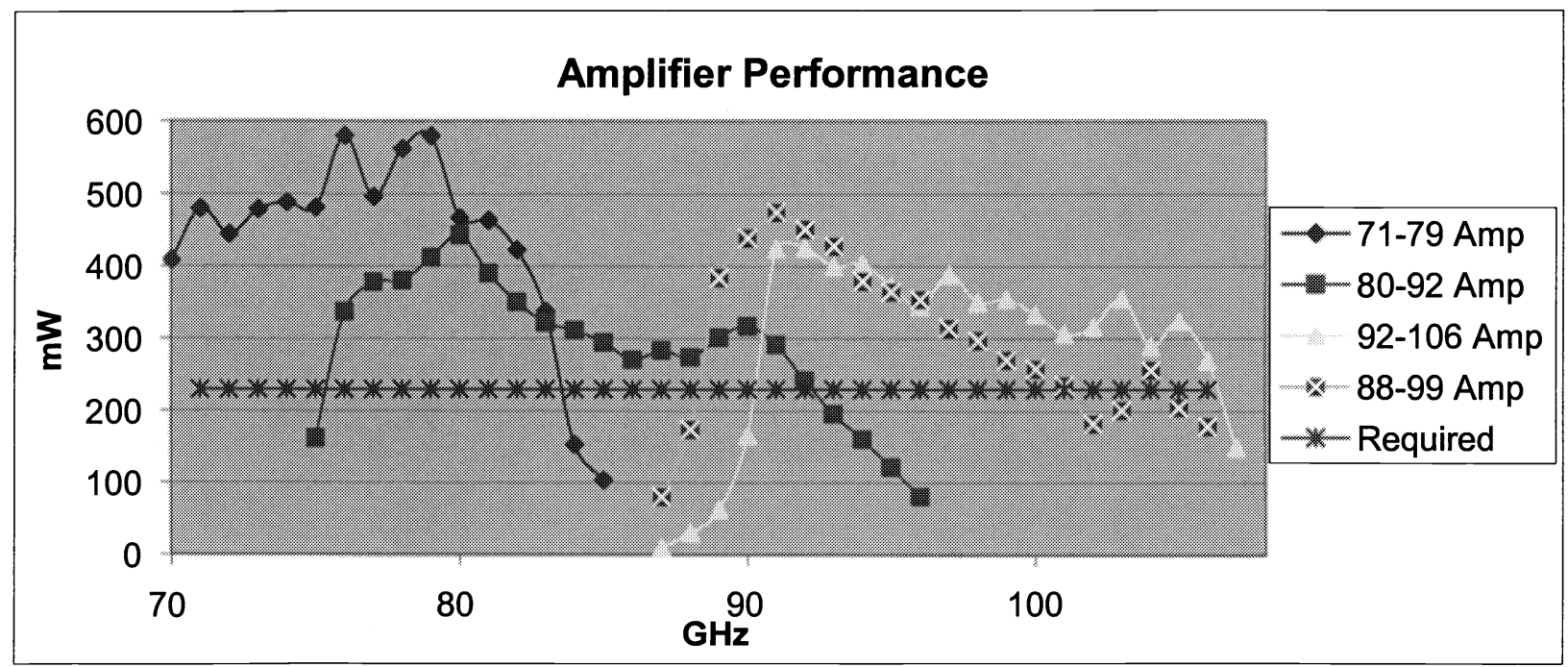

Figure 7. Typical output power of the JPL or TRW amplifier configuration when the output stages are well-matched for efficient power combining. 


\section{LOCAL OSCILLATORS}

At the time of the HIFI proposal there was no demonstrated local oscillator, besides a fixed frequency gas laser, which had sufficient CW power to pump an HEB mixers in HIFI band 6 . The situation was only marginally better for HIFI bands 1-5 where whisker contacted multipliers with input and output tuners had demonstrated up to $5 \%$ bandwidth and greater than a microwatt performance to $1350 \mathrm{GHz}^{34}$. The basic design was little changed from the original cross waveguide harmonic generator ${ }^{35}$ and due to challenges of whisker assembly could not really take advantage of symmetry and circuit balance to generate odd or even harmonics. Additionally, space qualification of whiskered devices has always been a difficult engineering task. For HIFI to be achievable in the time and budget available, the local oscillator concept needed a revolutionary approach. The one positive development prior to HIFI was the rapid development of computer power and electromagnetic simulation tools. These tools coupled with state of the art computers were capable of predicting the frequency response of microwave circuits with a very high degree of fidelity even at high frequency. Additionally, a number of complicated GaAs processing tricks had been developed for the processing of planar mixers $^{36,37}$ and high quality symmetric planar doublers had recently been demonstrated ${ }^{38}$. It appeared that the only viable approach was to go with all planar diodes since completely symmetric doublers and completely asymmetric triplers could be easily fabricated in a planar diode process, but not with a whiskered diode process. Planar devices also allowed tuning elements for de-imbedding the diode impedance to be placed close the diode without whisker dimensional constraints.

The individual HIFI multiplier stages needed to cover a range of output frequencies from 142 to $1910 \mathrm{GHz}$ and input powers from $400 \mathrm{~mW}$ to 500 microwatts. As a result, it was necessary to develop two processes. The first process is optimized for higher powers and lower-frequencies is done with a stepper lithography techniques for anode definition and is used for devices to roughly $1 \mathrm{THz}$. The second process uses e-beam lithography for anode definition and chemically etched membrane structures for devices above $1 \mathrm{THz}$. Both process can generate suspended metal structures, air bridges, beam leads and on chip capacitors ${ }^{39}$. Figure 8 shows the four basic types of devices that can be produced. These include the "discrete" devices, which are designed for flip chip soldering attachment, the "substrateless" devices which integrate the wiring with the diode for drop in installation without solder, the frameless membrane devices which also drop in and the framed membrane devices, which have a frame for handling.
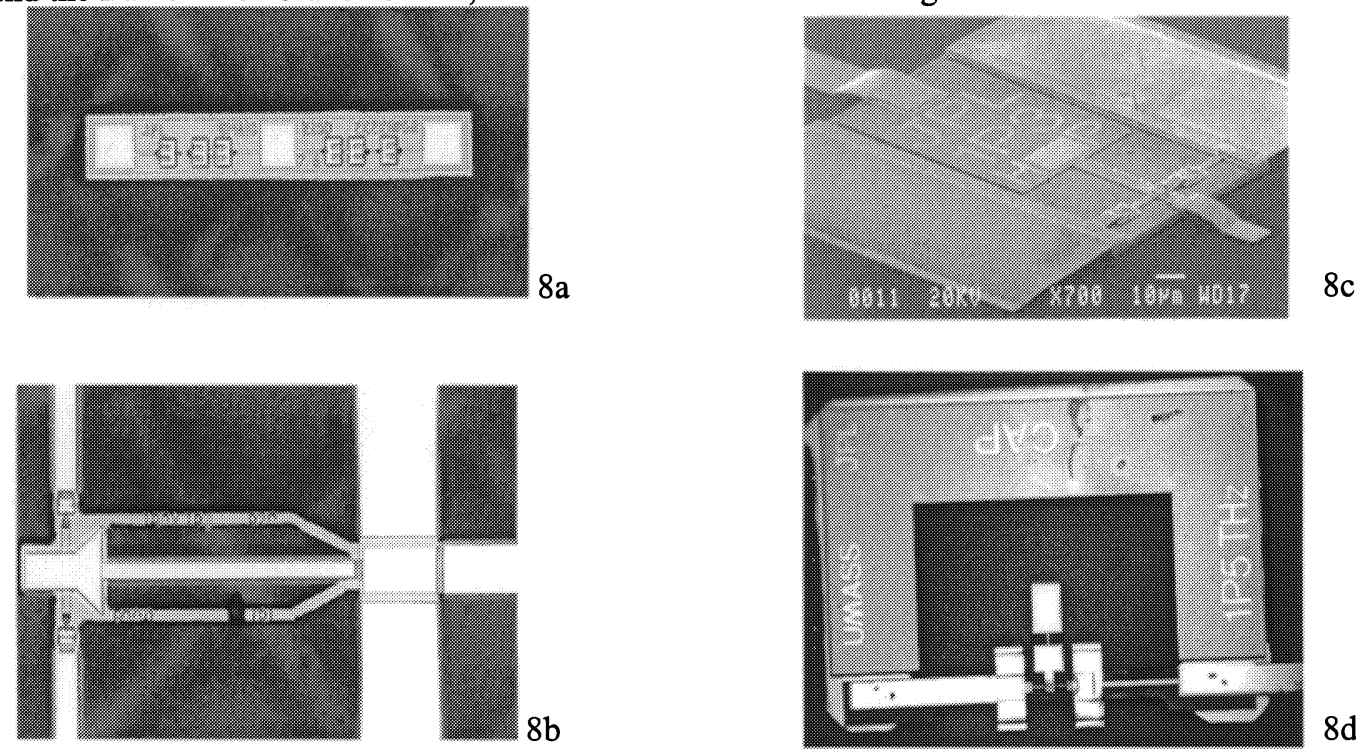

Figure 8a-d: Figure $8 \mathrm{a}$ a discrete diode used for a $200 \mathrm{GHz}$ doubler. Figure $8 \mathrm{~b}$ a substrateless diode used for an $800 \mathrm{GHz}$ doubler. Figure $8 \mathrm{c}$ a frameless membrane used for a $1200 \mathrm{GHz}$ tripler. Figure $8 \mathrm{~d}$ a framed membrane used in a $1.5 \mathrm{THz}$ doubler.

The planar devices with beam leads simplify greatly the assembly of the multiplier making the most challenging part of multiplier chain construction the fabrication of the waveguide circuit. The HIFI band 5 multiplier is a $\times 2 \times 2 \times 3$ configuration starting from 93.9 to $103.5 \mathrm{GHz}$. Figure 9 shows the development model chain in its as delivered 
configuration. It features a flexure mount, which enables the position of the output diagonal horn to be positioned to a few microns in two axes and better than 0.1 degrees in two angels. A machining tolerance of less than 0.0002 " ( 5 microns) has been achieved for the waveguide circuits and horns. Figure 10 shows the achievable performance for a $1200 \mathrm{GHz}$ chain with a constant $150 \mathrm{~mW}$ input power. This should be compared to the previous state of the art of $30 \mu \mathrm{W}$ peak and $\sim 2 \%$ bandwidth for a whiskered chain ${ }^{33}$. The planar construction has proven to be very robust surviving many thermal cycles and high random vibration levels. The only observed problems have been the result of over voltage or electrostatic discharge. Additional details on this multiplier chain have been presented previously ${ }^{40-42}$ and will be updated in paper $4855-56^{4}$.

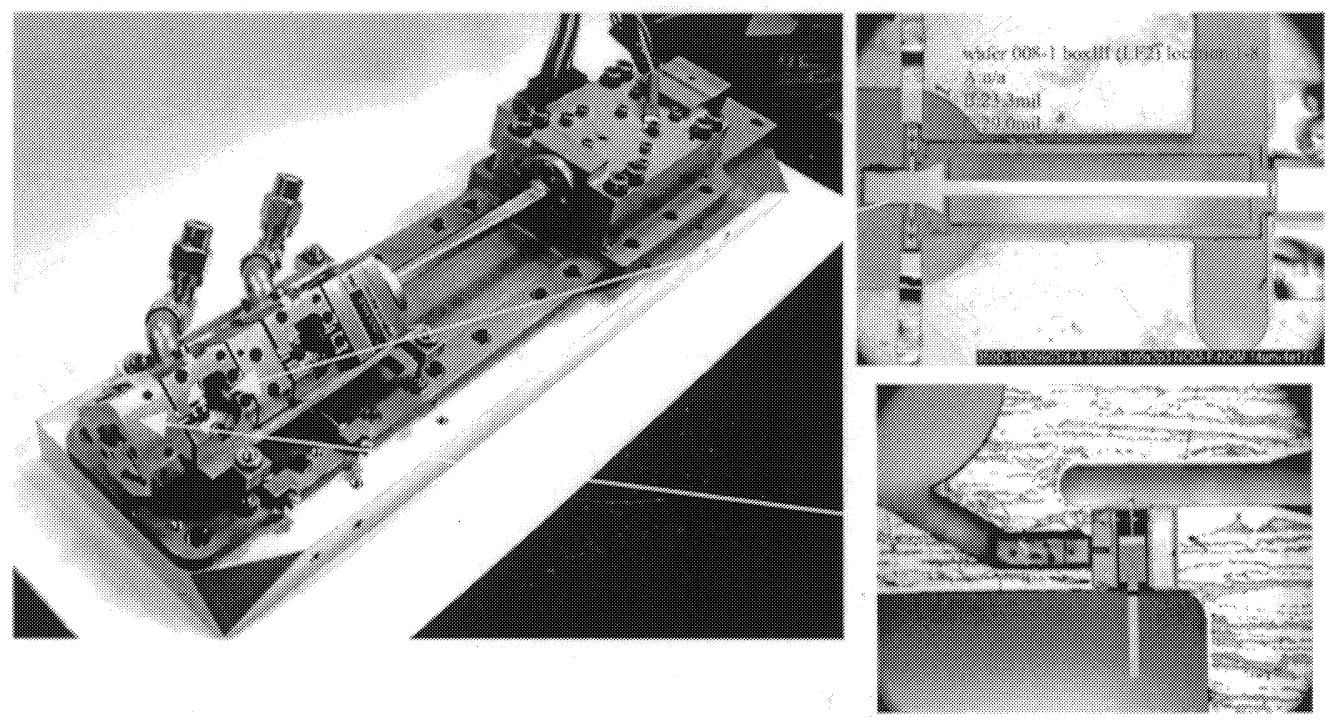

Figure 9. Development model $1200 \mathrm{GHz}$ multiplier chain. From left $1200 \mathrm{GHz}$ tripler with internal diagonal horn, $400 \mathrm{GHz}$ doubler with internal detail, space holder for WR-5 isolator, $200 \mathrm{GHz}$ doubler, WR-10 isolator, WR-10 waveguide twist, $92-106 \mathrm{GHz}$ power amplifier, input isolator on flexure mount. The two smaller pictures show the mounting of the $400 \mathrm{GHz}$ doubler diode and the 1200 $\mathrm{GHz}$ tripler diode in the waveguide channels.

\section{Band 5 LO Performance}

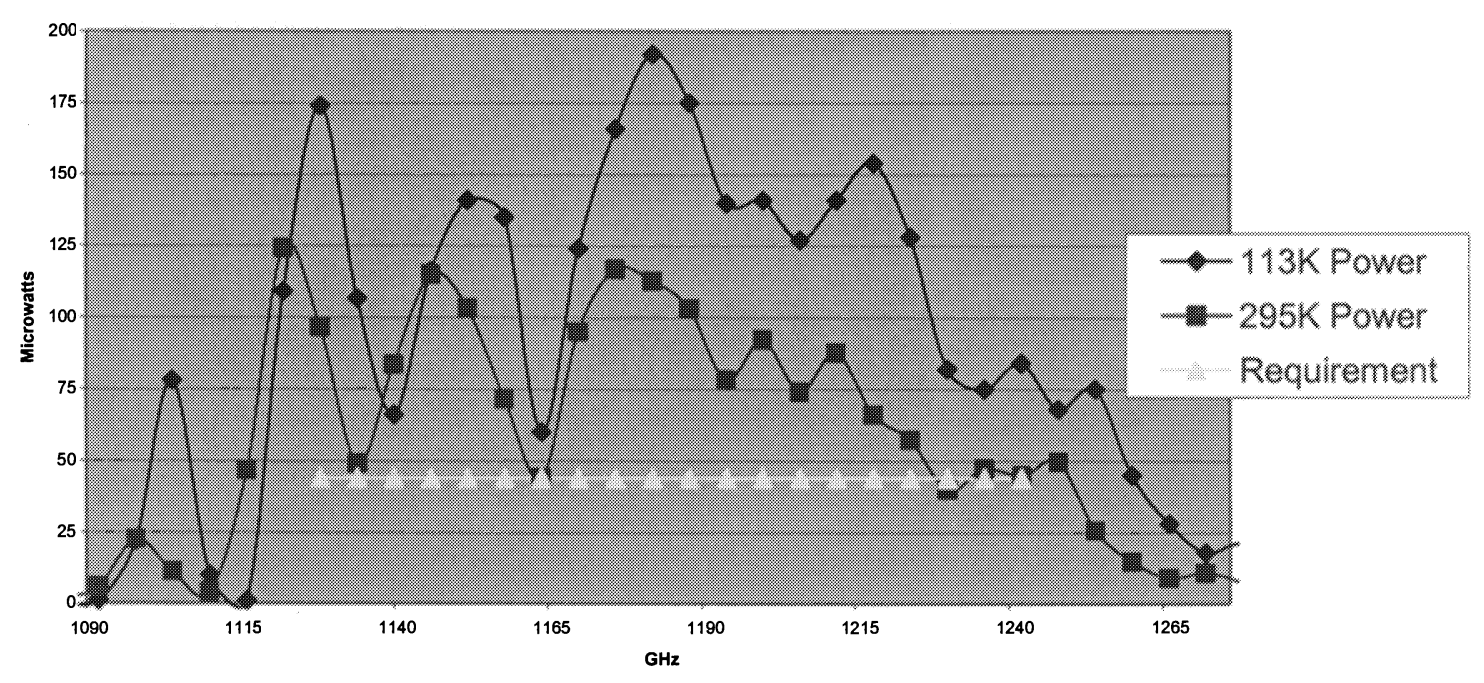

Figure 10 Performance of an all-planar all solid-state $1200 \mathrm{GHz}$ multiplier chain. The required power is at $120 \mathrm{~K}$ and covers $1127-$ $1242 \mathrm{GHz}$. It should be noted that two multiplier chains could be used to cover this band, but only one was used here with a flat $150 \mathrm{~mW}$ of input power. 
Local oscillators above $1.4 \mathrm{THz}$ required the largest advances in the state of the art at the start of the HIFI project. The physics of multiplier diodes at $\mathrm{THz}$ frequencies needed careful exploring, the technology for machining structures with dimensions on the order of 10 microns needed development and a process for assembling each new multiplier design needed development. Verification of results must contend with difficulties in measurement of microwatt power levels at $\mathrm{THz}$ frequencies, atmospheric absorption and cryogenic operation. Significant progress has been made in all these areas. A series of papers on the physics of multiplier diodes ${ }^{43-45}$ describes the diode parameters and modeling considerations for $\mathrm{THz}$ design. Machining tooling and processes have been developed and demonstrated to the necessary precision and the drop in assembly including handling of the very small frameless $\mathrm{THz}$ devices has proven to be feasible. The testing and verification area has explored the effects of cryogenic operation ${ }^{46-48}$ and tested multipliers to $2.7 \mathrm{THz}^{49,50}$, A number of designs and device topologies have also been tested with very promising results ${ }^{51-53}$. The most promising has been the development of a four-stage multiplication chain of cascaded doublers starting from $90-100 \mathrm{GHz}^{54}$. This chain is shown in Figure 11. Figure 12 shows the results at the various stages of multiplication for operation at $77 \mathrm{~K}$. Considerable effort is currently going into the design of the THz stages needed for 1.4 to $1.9 \mathrm{THz}^{55}$. Paper $4855-55^{3}$ updates the current status of THz multiplier design for Herschel HIFI.

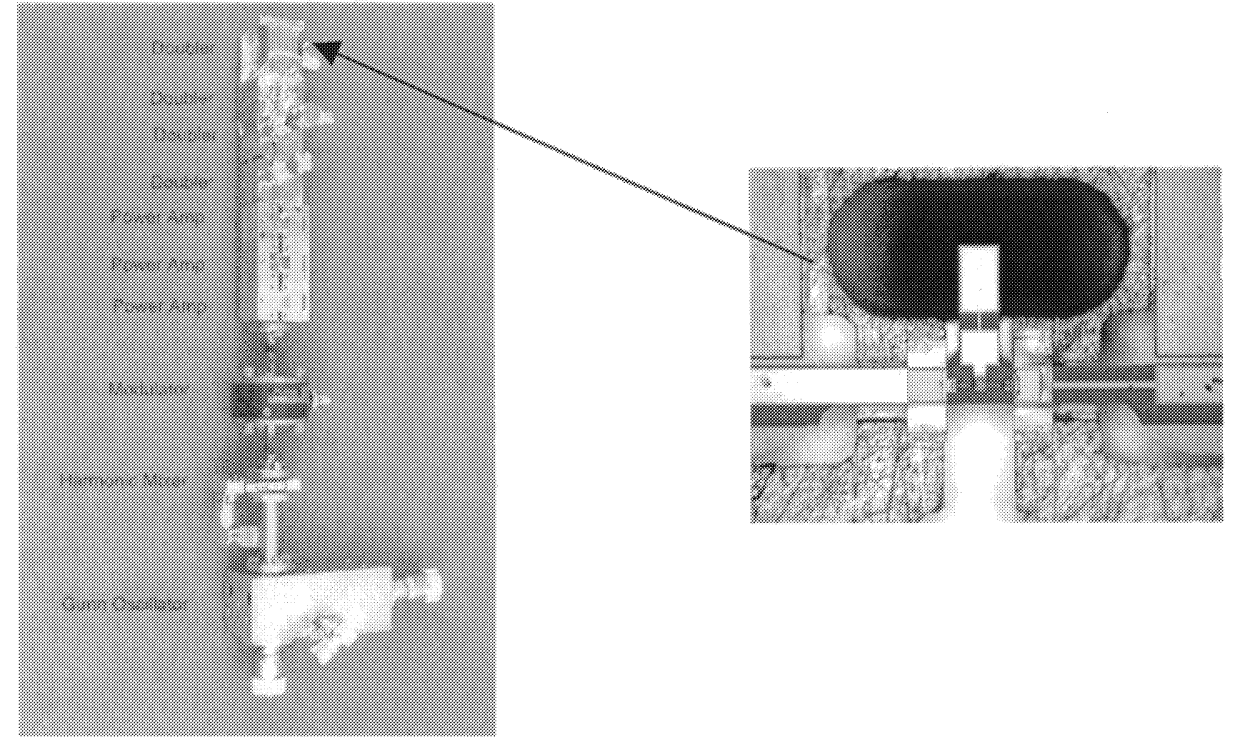

Figure 11. 1.5 THz all planar multiplier chain. Inset shows the framed membrane double in the final stage. The last two stages are coupled with a horn-to-horn interface.

\section{CONCLUSIONS}

The NASA sponsored high frequency component development for Herschel's HIFI instrument has resulted in the development and implementation of a wide range of new submillimeter technology. In the area of receivers, low noise SIS mixers can be made to over $1.2 \mathrm{THz}$, diffusion cooled Nb HEB mixers have demonstrated the necessary robustness for space application and InP based HEMT amplifiers with an octave bandwidth have less than $4 \mathrm{~K}$ noise temperatures. Multiplied local oscillators have been revolutionized by the use of high frequency, high power, broad banded amplifiers, planar diodes, membrane diodes and the level of circuit integration possible. Previously unimaginable power and bandwidth results have been reported simultaneously in the same multiplier chains. As a result of these THz component developments, the Herschel Space Observatories HIFI instrument is assured unprecedented frequency coverage and outstanding sensitivity for the benefit of the observation community.

\section{ACKNOWLEDGEMENT}

This research was carried out at the Jet Propulsion Laboratory, California Institute of Technology, under a contract with the National Aeronautics and Space Administration. 


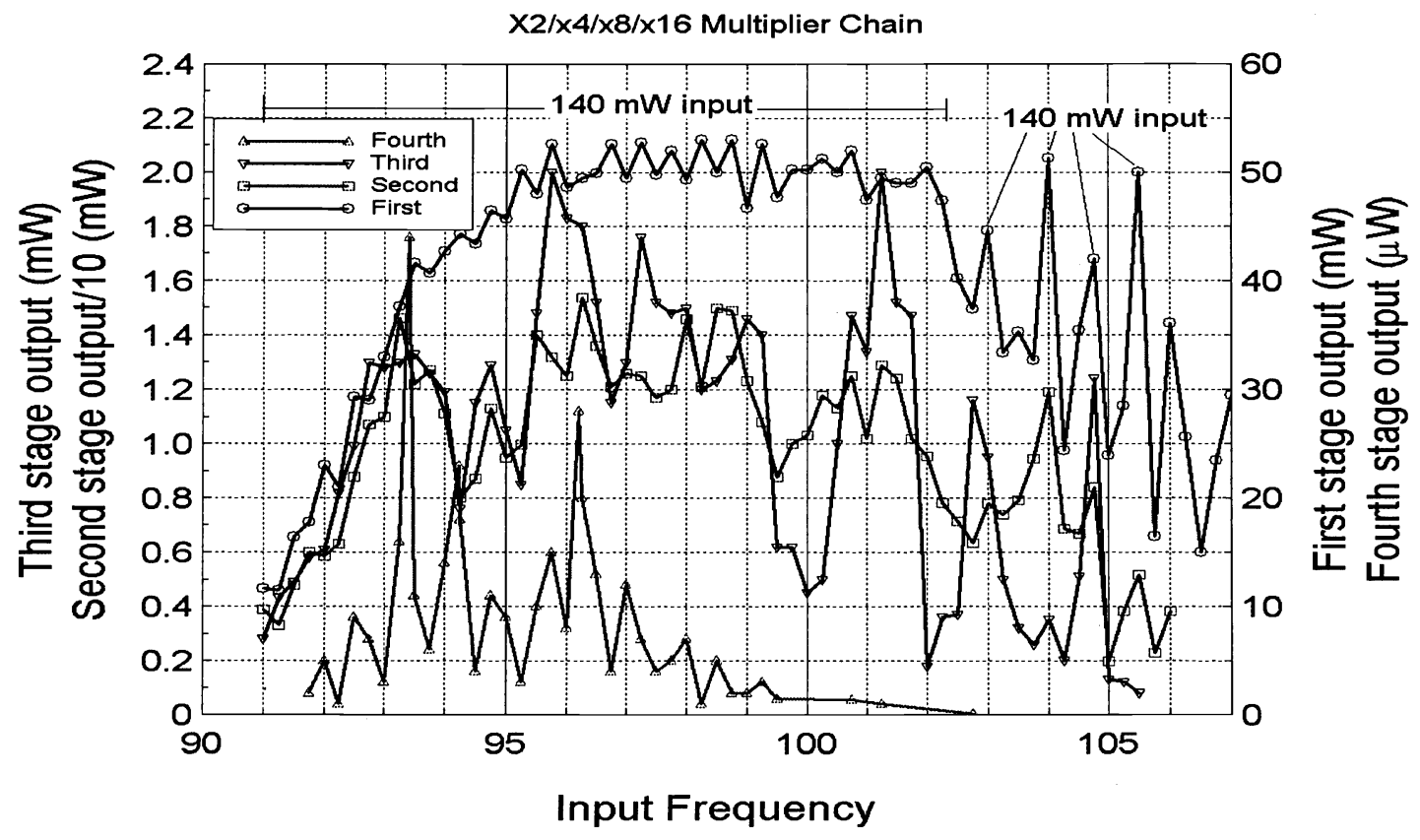

Figure 12. Measured 1.4-1.6 THz performance of the all-planar $1.5 \mathrm{THz}$ chain at $77 \mathrm{~K}$ with $140 \mathrm{~mW}$ input. HEB mixers require less than one microwatt to fully pump them. Assuming $3 \mathrm{~dB}$ of optical losses and dual polarization operation, this chain would cover about $100 \mathrm{GHz}$ of bandwidth.

\section{REFERENCES}

1. J. Kawamura, D.G. Harding, W.R. McGrath, B. Bumble, R. LeDuc \& P. Focardi, "1.8 THz superconductive hotelectron bolometer mixer for Herschel," Proc. SPIE vol. 4855, in Press, 2002.

2. A. Karpov, D. Miller, J. Zmuidzinas, J.A. Stern, B. Bumble, H.G. LeDuc, "1.2 THz SIS mixers for Herschel radio telescope," Proc. SPIE vol. 4855, in Press, 2002.

3. I. Mehdi, E. Schlecht, G. Chattopadhyay \& I. Mehdi, "THz Local Oscillator Sources," Proc. SPIE vol. 4855, in Press, 2002.

4. F. Maiwald, E. Schlecht, A. Maestrini, G. Chattopadhyay, D. Pukala, J.C. Pearson \& I. Mehdi, "Thz frequency multiplier chains based on planar Schottky diodes," Proc. SPIE vol. 4855, in Press, 2002.

5. R.R. Ferber, T.C.Gaier, J.C. Pearson, L.A. Samoska, A. Campbell, M. Wells, G. Swift, P. Yocom, \& Y. Chung, "WBand Power Amplifier Development for the Herschel HIFI Instrument," Proc. SPIE vol. 4855, in Press, 2002

6. C. Risacher \& V. Belitsky, "Low noise cryogenic IF-amplifiers for super heterodyne radioastronomy receivers," Proc. SPIE vol. 4855, in Press, 2002.

7. I. Lopez-Fernandez, J.D. Gallego-Puyol, C. Diez \& J. Martin-Pintado, "Wideband ultra-low noise cryogenic InP IF amplifiers for the Herschel mission radiometers," Proc. SPIE vol. 4855, in Press, 2002.

8. T. de Graauw, E. Caux, T.G. Phillips, J. Stutzki, N.D. Whyborn et al., "Herschel heterodyne instrument for the farinfrared (HIFI): overview and development," Proc. SPIE vol. 4850, in Press, 2002.

9. L. Ravera, Ph. Cais, M. Giard, A. Baudry, D. Lagrange, G. Montignac, J.L. Noullet, E. Caux, A. Cros, J.M. Desbats, J.B. Begueret, D. Navarro \& N. Lavigne, "A wide band, high resolution, versatile spectrometer proposed for FIRSTHIFI," Proc. SPIE vol. 4013, pp. 305-312, 2000.

10. R. Schieder, O. Siebertz, F. Schloeder, C. Gal, J. Stutzki, P. Hartogh \& V. Natale, "Wide-Banded Spectrometer for HIFI-FIRST," Proc. SPIE vol. 4013, pp. 313-324, 2000.

11. J.C. Pearson, R. Gueston, T. Klein \& N.D. Whyborn, "Local Oscillator System for the heterodyne instrument for FIRST (HIFI)," Proc. SPIE vol. 4013, pp. 264-274, 2000. 
12. T. Klein, R. Gueston, C. Kasemann, P. Orleanski, T. Rose, S.D. Philipp, A. Wunsch, "Local Oscillator Unit for Herschel/HIFI," Proc. SPIE vol. 4850, in press, 2002.

13. P. Dieleman, T.M. Klapwijk, J. R. Gao \& H. van de Stadt, "Analysis of $\mathrm{Nb}$ superconductor-insulatorsuperconductor tunnel junctions with $\mathrm{Al}$ striplines for THz radiation detection," IEEE Trans. Appl. Supercond. 7, pp. 2566-2569, 1997.

14. M. Bin, M.C. Gaidis, J. Zmuidzinas, T.G. Phillips, \& H.G. Leduc, "Low-noise $1 \mathrm{THz}$ niobium superconducting tunnel junction mixer with a normal metal tuning ciruit," Appl. Phys. Lett. 68, pp. 1714-1716, 1996.

15. J. Kawamura, R. Blundell, C.-Y. E. Tong, G. Gol'tsman, E. Gershenzon \& B. Voronov, "Performance of NbN lattice-cooled hot-electron bolometric mixers," J. Appl. Phys. 80, pp. 4232-4234, 1996.

16. H. Ekxtrom, E. Kollberg, P. Yagoubov, G. Gol'tsman, E. Gershenzon \& S. Yngvesson, "Gain and noise bandwidth of NbN hot-electron bolometric mixers," Appl. Phys. Lett. 70, pp. 3296-3298, 1997.

17. A. Skalare, W.R. McGrath, B. Bumble, H.G. LeDuc, P.J. Burke, A.A. Verheijen, R.J. Schoelkopf \& D.E. Prober, "Large bandwidth and low noise in a diffusion-cooled hot-electron bolometer mixer." Appl. Phys. Lett. 68, pp1558$1660,1996$.

18. J. Kawamura, J. Chen, D. Miller, J. Kooi, J. Zmuidzinas, B. Bumble, H.G. LeDuc \& J.A. Stern, "Low-noise submillimeter-wave NbTiN superconducting tunnel junction mixers," Appl. Phys. Lett. 75, pp.4013-4015, 1999.

19. J.W. Kooi, J. Kawamura, J. chen, G. Chattopadhyay, J.R. Pardo, J. Zmuidzinas, T.G. Phillips, B. Bumble, J. Stern \& H.G. LeDuc, "A Low Noise NbTiN-based $850 \mathrm{GHz}$ SIS Receiver for the Caltech Submillimeter Observatory," Int. J. Infrared \& MM. Waves 21, pp. 689-716, 2000.

20. J. Kawamura, D. Miller, J. Chen, J. Zmuidzinas, B. Bumble, H.G. LeDuc \& J.A. Stern, "Very high-current-density $\mathrm{Nb} / \mathrm{AlN} / \mathrm{Nb}$ tunnel junctions for low-noise submillimter mixers," Appl. Phys. Lett. 76, pp.2119-2121, 2000.

21. A. Karpov, J. Zmuidzinas, F. Rice, D. Miller, J.A. Stern, B. Bumble \& H.G. LeDuc, "Low Noise SIS mixer with Nb Tuning Circuit for the Frequency above $1 \mathrm{THz}$," Proc. $13^{\text {th }}$ Int. Symp. Space THz Tech. In press, 2002.

22. A. Skalare, W.R. McGrath, P.M. Echternach, H.G. LeDuc, I. Siddiqi, A. Verevkin \& D.E. Prober, "Aluminum HotElectron Bolometer Mixers at Submillimeter Wavelengths," IEEE Trans. Appl. Supercond. 11, pp. 641-644, 2001.

23. I. Siddiqi, A. Verevkin, D.E. Prober, A. Skalare, B.S. Karasik, W.R. McGrath, P.M. Echternach \& H.G. LeDuc, "Noise and Conversion Efficiency of Aluminum Superconducting Hot-Electron Bolometer Mixer," IEEE Trans. Appl. Supercond. 11, pp. 958-961, 2001

24. I. Siddiqi, A. Verevkin, D.E. Prober, A. Skalare, W.R. McGrath, P.M. Echternach \& H.G. LeDuc, "Heterodyne mixing in diffusion-cooled superconducting aluminum hot-electron bolometers," J. Appl. Phys. 91, pp. 4646-4654, 2002.

25. R.A. Wyss, B.S. Karasik, W.R. McGrath, B. Bumble \& H.G. LeDuc, “"” Proc. $10^{\text {th }}$ Int. Symp. Space THz Tech., pp. 215-228, 1999.

26. R.A. Wyss, A. Neto, W.R. McGrath, B. Bumble \& H.G. LeDuc, "Submillimeter-wave spectral response of twin-slot antennas coupled to hot electron bolometers," Proc. $11^{\text {th }}$ Int. Symp. Space THz Tech., pp. 379-388, 2000.

27. P. Focardi, A. Neto \& W.R. McGrath, "Accurate embedding circuit model for terahertz HEB receivers," Proc. IEEE Antennas \& Propagation Society Int. Symp. 2002, pp. 356-359, 2002.

28. P.-P. Huang, T.-W. Huang, H. Wang, E.W. Lin, Y. Shu, G.S. Dow, R. Lai, M. Biedenbender \& J.H. Elliot, "A 94GHz 0.35 W Power Amplifier Module," IEEE Trans. Microwave Theory and Tech. 45, pp 2418-2423, 1997.

29. T. Gaier, I. Mehdi, J. Kooi, B. Fujiwara \& R. Lai, "A W-band HEMT based power amplifier module for millimeterwave LO multipliers," Proc. SPIE Vol. 3357, pp 114-121, 1998.

30. L. Samoska, T. Gaier, A. Peralta, S. Weinreb, J. Bruston, I. Mehdi, Y.C. Chen, H.H. Liao, M. Nishimoto, R. Lai, H. Wang \& Y.C. Leong, "MMIC Power Amplifiers as Local Oscillator Drivers for FIRST," Proc. SPIE vol. 4013, pp 275-284, 2000.

31. H. Wang, L. Samoska, T. Gaier, A. Peralta, H.-H. Liao, Y.C. Leong, S. Weinreb, Y.C. Chen, M. Nishimoto \& R. Lai, "Power-Amplifier Modules Covering 70-113 GHz Using MMICs," IEEE Trans. Microwave Theory and Tech. 49, pp. 9-16, 2001.

32. H. Wang, L. Samoska, T. Gaier, A. Peralta, H.-H. Liao, Y.C. Chen, M. Nishimoto \& R. Lai, "Monolithic Power Amplifiers covering 70-113 GHz,” Proc. IEEE RF. Integrated Circuit Symp., pp 39-42, 2000.

33. L. Samoska, Kun-You Lin, Huei Wang, Yun-Ho Chung, Michael Aust, Sander Weinreb, and Douglas Dawson, "On the stability of Millimeter-Wave Power Amplifiers," IEEE International Microwave Symposium Digest, Vol. 1, pp.429-432, 2002.

34. P. Zimmernann, "Multipliers for THz Local Oscillators," Proc. SPIE vol. 3357, pp. 152-158, 1998. 
35. W.C. King \& W. Gordy, "One-to-two Millimeter wave spectroscopy. IV Experimental Methods and Results for OCS, CH3F and H2O," Phys. Rev. 93, pp. 407-412, 1954.

36. R.P. Smith, S.C. Martin, M. Kim, J. Bruston, D. Humphrey \& P.H. Siegel, "Advances in Submillimter Semiconductor-based Device Designes and Processes," Proc. $8^{\text {th }}$ Int. Symp. Space THz Tech. pp. , 1997.

37. P.H. Siegel, R.P Smith, M.C. Gaidis \& S.C. Martin, " $2.5 \mathrm{THz}$ GaAs monolithic membrane-diode mixer," IEEE Trans. Microwave Theory and Tech. 47, pp 596-604, 1999.

38. J. Tuovinen \& N.R. Erickson, "Analysis of a 170-GHz Frequency doubler with an array of planar diodes," IEEE Trans. Microwave Theory and Tech. 43, pp. 962-968, 1995.

39. S. Martin, B. Nakamura, A. Fung, P. smith, J. Bruston, A. Maestrini, F. Maiwald, P. Siegel, E. Schlecht \& I. Mehdi, "Fabrication of 200 to $2700 \mathrm{GHz}$ Multiplier Devices using GaAs and Metal Membranes," Microwave Symp. Digest 2001 IEEE MTT-S Int. Vol. 3., pp 1641-1644, 2001.

40. E. Schlecht, G. Chattopadhyay, A. Maestrini, A. Fung, S. Martin, D. Pukala, J. Bruston \& I. Mehdi, " 200,400 and $800 \mathrm{GHz}$ Schottky diode "substrateless" multipliers: design and results," Microwave Symp. Digest 2001 IEEE MTT-S Int. Vol. 3., pp 1649-1652, 2001.

41. A Maestrini, J. Bruston, D. Pukala, S. Martin \& I. Mehdi, "Performance of a $1.2 \mathrm{THz}$ frequency tripler using a GaAs frameless membrane monolithic circuit.” Microwave Symp. Digest 2001 IEEE MTT-S Int. Vol. 3., pp 1657-1660.

42. J. Bruston, A. Maestrini, D. Pukala, S. Martin, B. Nakamura \& I. Mehdi, "A $1.2 \mathrm{THz}$ planar tripler using GaAs membrane based chips," Proc. 12 ${ }^{\text {th }}$ Int. Symp. Space THz Tech., pp. 310-319, 2001.

43. J. East \& I. Mehdi, "Temperature effects in varactors and multipliers," Proc. $12^{\text {th }}$ Int. Symp. Space THz Tech., pp. 340-351, 2001.

44. E. Schlecht, F. Maiwald, G. Chattopadhyay, S. Martin, \& I. Mehdi, "Design Considerations for Heavily-Doped Cryogenic Schottky Diode Varactor Multipliers," Proc. 12 ${ }^{\text {th }}$ Int. Symp. Space THz Tech., pp. 485-494, 2001.

45. E. Schlecht, G. Chattopadhyay, A. Maestrini, D. Pukala, J. Gill \& I. Mehdi, "Harmonic Balance Optimization of Terhertz Schottky Diode Multipliers Using an Advanced Device Model," Proc. $13^{\text {th }}$ Int. Symp. Space THz Tech., in press, 2002.

46. E. Schlecht, J. Bruston, A. Maestrini, S. Martin, D. Pukala, R. Tsang, A. Fung, R.P. Smith \& I. Mehdi, "200 and 400 $\mathrm{GHz}$ Schottky diode multipliers fabricated with integrated air dielectric "substrateless" circuitry," Proc $11^{\text {th }}$ Int. symp. Space THz Tech., pp 287-295, 2000.

47. N.R. Erickson, G. Narayanan, R.P. Smith, S.C. Martin, I. Mehdi, T.W. Crowe \& W.L. Bishop, "Planar Frequency Doublers and Triplers for FIRST," Proc $11^{\text {th }}$ Int. symp. Space THz Tech., pp 543-551, 2000.

48. A. Maestrini, D. Pukala, F. Maiwald, E. Schlecht, G. Chattopadhyay \& I. Mehdi, "Cryogenic operation of GaAs based multiplier chains to $400 \mathrm{GHz}$," Proc. $8^{\text {th }}$ Int. THz Conference, 2000.

49. F. Maiwald, S. Martin, J. Bruston, A. Maestrini, T. Crawford \& P.H. Siegel, "2.7 THz waveguide tripler using monothlithic membrane diodes," Microwave Symp. Digest 2001 IEEE MTT-S Int. Vol. 3., pp 1637-1640, 2001.

50. F. Maiwald, S. Martin, J. Bruston, A. Maestrini, T. Crawford \& P.H. Siegel, "Design and performance of a $2.7 \mathrm{THz}$ waveguide tripler," Proc $12^{\text {th }}$ Int. symp. Space THz Tech., pp. 320-329, 2001.

51. G. Chattopadhyay, E. Schlecht, J. Gill, S. Martin, A. Maestrini, D. Pukala, F. Maiwald \& I. Mehdi, "A broadband $800 \mathrm{GHz}$ Schottky balanced doubler," IEEE Microwave and Wireless Component Lett. 12, pp. 117-118, 2002.

52. High Efficieny MMIC triplers for Millimter and Submillimeter wavelengths," Microwave Symp. Digest 2000 IEEE MTT-S Int. Vol. 3., pp 1003-1006, 2000.

53. N.R. Erickson, G. Narayanan, R.P. Smith, S.C. Martin, I. Mehdi, M. Coulomb \& G. DeMartinez, "Monolitic Frequency Doublers and Triplers for THz Frequencies," Proc $12^{\text {th }}$ Int. symp. Space THz Tech., pp. 297-309, 2001.

54. N.R. Erickson, A. Maestrini, E. Schlecht, G. Chattopadhyay, J. Gill \& I. Mehdi, "1.5 THz all-planar Multiplied Source," Proc $13^{\text {th }}$ Int. symp. Space THz Tech., in press, 2002.

55. A. Maestrini, G. Chattopadhyay, E. Schlecht \& I. Mehdi, "1400-1900 GHz Membrane Based Schottly Diode Triplers," Proc $13^{\text {th }}$ Int. symp. Space THz Tech., in press, 2002. 